Research Article

\title{
On the Shape Simulation of Aggregate and Cement Particles in a DEM System
}

\author{
Huan He, ${ }^{1,2}$ Piet Stroeven, ${ }^{2}$ Eric Pirard, ${ }^{3}$ and Luc Courard ${ }^{3}$ \\ ${ }^{1}$ College of Architecture and Civil Engineering, Beijing University of Technology, Beijing 100122, China \\ ${ }^{2}$ Faculty of Civil Engineering and Geosciences, Delft University of Technology, Stevinweg 1, 2628 CN Delft, Netherlands \\ ${ }^{3}$ GeMMe (Minerals Engineering, Materials and Environment), University of Liège, Chemin des Chevreuils 1, 4000 Liège, Belgium
}

Correspondence should be addressed to Huan He; h.he@live.com

Received 30 April 2015; Revised 6 July 2015; Accepted 7 July 2015

Academic Editor: Ana S. Guimarães

Copyright (C) 2015 Huan He et al. This is an open access article distributed under the Creative Commons Attribution License, which permits unrestricted use, distribution, and reproduction in any medium, provided the original work is properly cited.

\begin{abstract}
Aggregate occupies at least three-quarters of the volume of concrete, so its impact on concrete's properties is significant. Both size and shape of aggregate influence workability, mechanical properties, and durability of concrete. On the other hand, the shape of cement particles plays also an important role in the hydration process due to surface dissolution in the hardening process. Additionally, grain dispersion, shape, and size govern the pore percolation process that is of crucial importance for concrete durability. Discrete element modeling (DEM) is commonly employed for simulation of concrete structure. To be able to do so, the assessed grain shape should be implemented. The approaches for aggregate and cement structure simulation by a concurrent algorithm-based DEM system are discussed in this paper. Both aggregate and cement grains were experimentally analyzed by $\mathrm{X}$-ray tomography method recently. The results provide a real experimental database, for example, surface area versus volume distribution, for simulation of particles in concrete technology. Optimum solutions are obtained by different simplified shapes proposed for aggregate and cement, respectively. In this way, more reliable concepts for aggregate structure and fresh cement paste can be simulated by a DEM system.
\end{abstract}

\section{Introduction}

A particle is defined as the smallest discrete unit of a powder mass that cannot be easily subdivided [1]. The shapes of real particles are irregular from coarse aggregate to a mineral admixture in concrete. Aggregate occupies at least threequarters of the volume of concrete, so its impact on concrete's properties is significant. The sieve curve traditionally defines the aggregate size range. Another essential property is grain shape. Both size and shape influence compactability and workability in the fresh state and the mechanical and durability properties of the hardened concrete. The definition of the actual grain shape as well as the representation in a discrete element modeling (DEM) system is complicated, however. Furthermore, the real shape of aggregate or binder particles can vary widely. The sphere is therefore generally adopted in conventional simulation systems in concrete technology that are mostly of random sequential addition (RSA) nature, despite imposing serious limitations and biases.
The shape of cement particles plays also an important role in the hydration process due to surface dissolution in the hardening process. Additionally, grain dispersion, shape, and size govern the pore percolation process that is of crucial importance for concrete durability [2]. Cement hydration in a DEM approach renders the possibility of investigating microstructure development and resulting properties of concrete. A digital image-based model [3] as well as continuum models [4-6] has been developed for this purpose. Apart from the digital image-based model, particle shape is generally assumed to be spherical, because of inherent simplification in algorithm formulation, however, at the cost of biases in the simulation results. Recently, some reference cement and aggregate materials were analyzed by microtomography $(\mu \mathrm{CT})$ and X-ray tomography $(\mathrm{CT})$, respectively $[7,8]$. The results provide real experimental databases of aggregate and cement grains, providing valuable parameters for the DEM simulation. This is even more so, since traditional shape analysis methods, for example, manual measurement or 


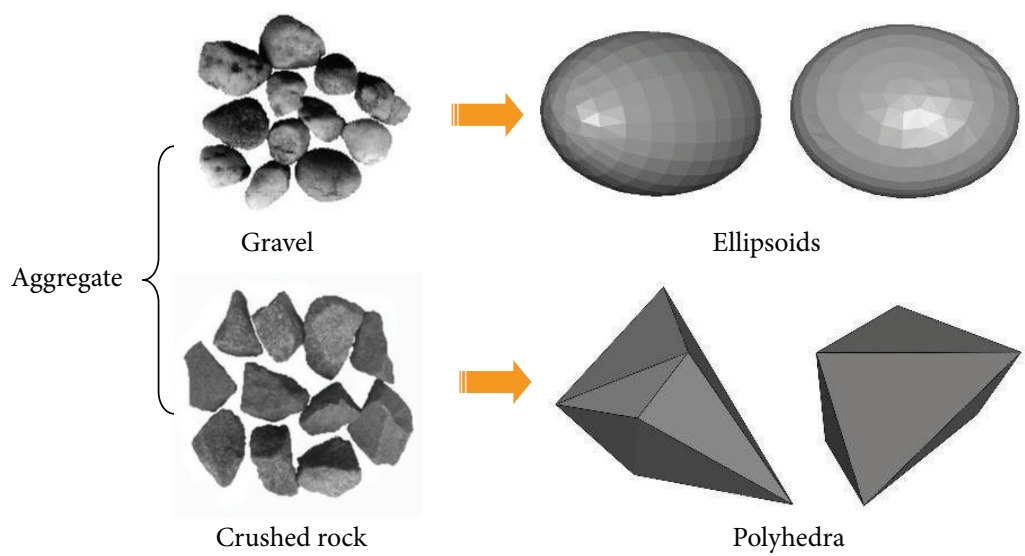

FIGURE 1: Simulation strategy of arbitrary shaped aggregate: (top) differently shaped ellipsoids represent river gravel; (bottom) differently shaped polyhedrons represent crushed rock.

experimental techniques in building codes such as ASTM D-3398-97, cannot provide sufficient information for particle reconstruction in the DEM systems. The involved experimental difficulties would be even greater for very fine particles such as cement grains. Simulation of concrete by a DEM system requires a large number of particles with different sizes to guarantee the RVE demand. They will inevitably also have different shapes. However, giving all particles individual shapes in DEM would be too expensive and (computer) time-consuming for solving practical engineering problems [9]. Therefore, a practical strategy is to select a single shape parameter, or eventually a limited number of typical shapes to represent a particles mixture.

In this paper, simulation strategies are presented for the representation of real aggregate and cement grains in concrete technology. This simulation approach is incorporated in a physical concurrent algorithm-based DEM system, that is, HADES. The system is developed for making more realistic packing simulations by using nonspherical particles. It is based on a contact mechanism that evaluates the interaction forces exerted between segmented surfaces of neighboring particles. It can provide packing densities up to the dense random packing state relevant for the present purposes. More detailed information about the system can be found in [10]. A practical shape analysis study is conducted on the basis of $\mathrm{CT}$ and $\mu \mathrm{CT}$ results available in the international literature $[7,8]$. Specific simulation strategies could be proposed for both aggregate and cement particle simulation.

\section{Shape Simulation of Aggregate Grains}

Aggregate in concrete can be divided into two groups, that is, gravel of fluvial origin and crushed rock, representing rounded and angular-shaped particles, respectively, as shown in Figure 1. The ellipsoid was selected to represent the river gravel particles, whereas the polyhedron was used for simulation of crushed rock grains. The surface of these model particles is tessellated by a mesh positioned on the grains' surfaces. The mesh should be fine enough for a proper generation of the particles and interparticle computational requirement [10].

As a practical example, a series of regular polyhedrons with 4 to 8 facetted surfaces were employed to represent crushed rock aggregate as used in railroad beds [11]. This greatly simplifies the simulation, since only the two parameters should be specified, that is, the sieve size and the maximum size of the surface element. Figure 2 presents the meshed particles of the regular polyhedrons employed in this study. The volume fraction of each type of polyhedron is taken similar to that found in the field study of Guo [11].

Recently, in a study of Erdogan et al. [8], the shapes of four different types of aggregate, denoted by GR, LS, IN, and AZ, were investigated by the CT method and reconstructed by the spherical harmonic $(\mathrm{SH})$ technique [8]. The most interesting information coming from this shape analysis is the global surface area $(S)$ to volume $(V)$ curve of each type of aggregate. A function $S=a V^{b}$ was used to represent the $S$ versus $V$ curve for each type of aggregate [8]. The coefficients $a$ and $b$ were assessed for all cases and are listed in Table 1 [8]. The variances of the regression approximations were exceeding 0.98 in all cases.

The regression function $S=a^{\prime} V^{2 / 3}$ was employed to fit the different types of aggregate. Next, $a^{\prime}$ is determined (volume $<4000 \mathrm{~mm}^{3}$ ) with a variation lower than $5 \%$ for each type of aggregate, as given in Table 1 . A common shape index like sphericity is finally determined for each type of aggregate by

$$
\text { Sphericity }=\frac{S_{\text {eq.sphere }}}{S_{\text {particle }}}=\frac{4 \pi(3 V / 4 \pi)^{2 / 3}}{a^{\prime} V^{2 / 3}}=4.836\left(a^{\prime}\right)^{-1}
$$

in which $a^{\prime}$ is the coefficient in the above-mentioned relationship of $S$ versus $V^{2 / 3}$. Sphericity is defined as the surface area ratio of the equivalent sphere and the real particle, so both having equal volume. The sphericity values are also listed in Table 1. Obviously, the crushed rock types GR and LS cannot be distinguished by means of sphericity. The natural river gravel types IN and AZ have a relatively larger sphericity, as 


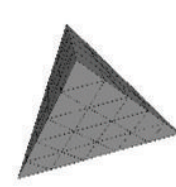

Tetrahedron
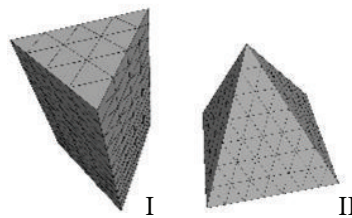

Pentahedron

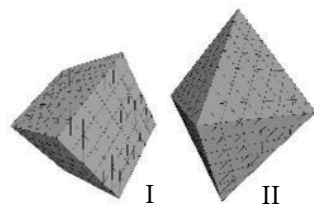

Hexahedron
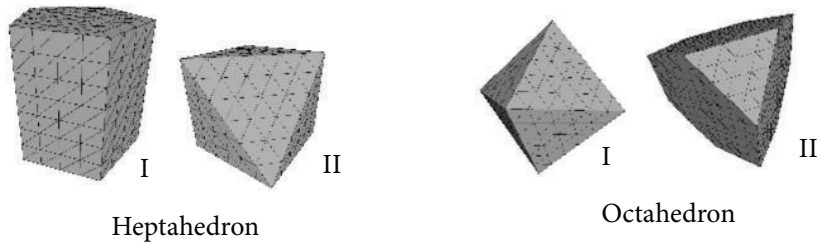

Octahedron

FIGURE 2: Nine regular polyhedra with facet numbers $4 \sim 8$.

TABLE 1: $S-V$ correlation coefficients found by Erdogan et al. [8] for four types of aggregate: $a$ and $b$ are from $S=a V^{b}$, while coefficient $a^{\prime}$ is from $S=a^{\prime} V^{2 / 3}$. Sphericity is calculated by (1).

\begin{tabular}{|c|c|c|c|c|c|}
\hline Aggregate type & Coefficient $a$ & Coefficient $b$ & Variance $R^{2}$ & Coefficient $a^{\prime}$ & Sphericity \\
\hline GR & 8.1 & 0.63 & 0.99 & 6.13 & 0.79 \\
\hline LS & 7.5 & 0.64 & 0.997 & 6.12 & 0.79 \\
\hline IN & 8.6 & 0.62 & 0.99 & 6.06 & 0.80 \\
\hline $\mathrm{AZ}$ & 9.1 & 0.61 & 0.98 & 5.95 & 0.81 \\
\hline
\end{tabular}

TABLE 2: Shape indices of the regular polyhedra, appropriate proportions according to Guo [11], and revised proportions for better overall simulation results.

\begin{tabular}{lccccc}
\hline Shapes & Facet number & Sphericity & $a^{\prime}$ & Proportion by Guo [11] & Revised proportion \\
\hline Tetrahedron & 4 & 0.67 & 7.21 & $10.0 \%$ & $5 \%$ \\
Pentahedron I & 5 & 0.70 & 6.95 & $12.5 \%$ & $5 \%$ \\
Pentahedron II & 5 & 0.72 & 6.71 & $12.5 \%$ & $5 \%$ \\
Hexahedron I (cube) & 6 & 0.81 & 6.00 & $17.5 \%$ & $10 \%$ \\
Hexahedron II & 6 & 0.76 & 6.39 & $17.5 \%$ & $10 \%$ \\
Heptahedron I & 7 & 0.83 & 5.83 & $10.0 \%$ & $15.5 \%$ \\
Heptahedron II & 7 & 0.80 & 6.06 & $5.0 \%$ & $15.5 \%$ \\
Octahedron I & 8 & 0.85 & 5.72 & $5.0 \%$ & $17 \%$ \\
Octahedron II & 8 & 0.78 & 6.24 & - & - \\
Sphere & $\infty$ & 1.00 & 4.84 & & $17 \%$ \\
\hline
\end{tabular}

could be expected. But sphericity seems not very effective in distinguishing between actual shapes.

The proposed proportions by Guo [11] for simulation of crushed rock in railway beds are then analyzed on the basis of experimental results of Erdogan et al. [8]. Shape indices of different regular polyhedra are listed in Table 2 with the sphere as a reference. It demonstrates that the shape indices of crushed rock samples GR and LS in Table 1 are within the range of shape indices of these nine regular polyhedra. Guo [11] also proposed for the crushed rock proportions of each shape category, as listed in Table 2. Next, the composite polyhedra method proposed by Guo [11] can be compared with experimental results of GR and LS [8], shown in Figure 3. Generally speaking, Guo's proposal fits the data of aggregates GR and LS quite well. But there is some deviation in the large particle range. Therefore, a better proportion of each category is indicated in Table 1 as well as in Figure 3. By theses proportions, $S-V$ information of composite polyhedra can better comply with the experimental results of GR and LS types. This practical example on crushed rock in railway beds shows that a proper global representation can be obtained by the sketched simple simulation strategy.

On the other hand, ellipsoids are particularly interesting because with only 3 parameters a variety of shapes, ranging from oblate to oblong, can be described. Three principal axes ( $a$ represents the longest, $b$ the medium size, and $c$ 


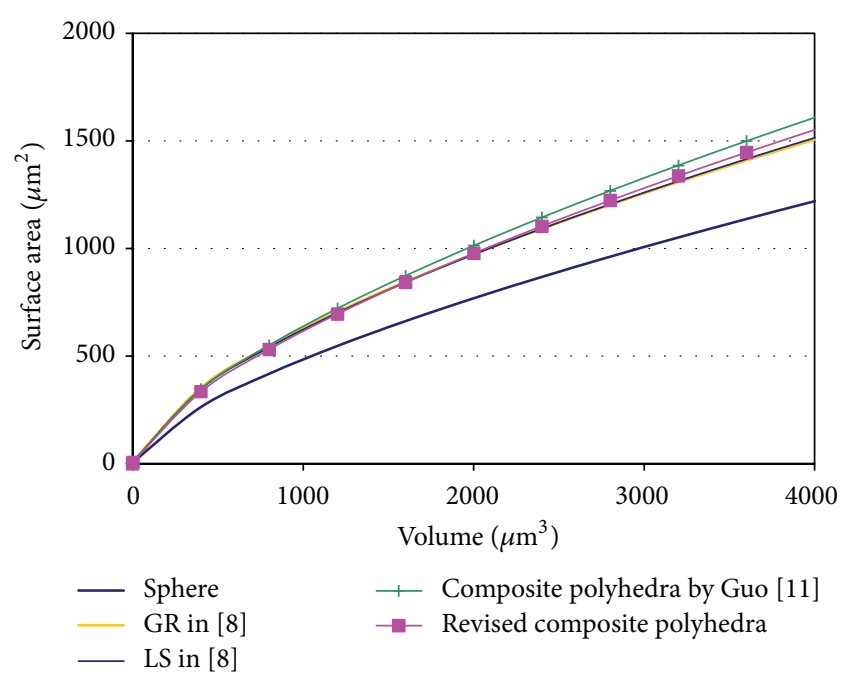

FIGURE 3: $S-V$ curves of crushed rock types (GR and LS), of composite polyhedra and of the sphere.

the shortest axis) determine the shape of an ellipsoid. By parameter variation, different ellipsoids with different $S-V$ relationships can be derived. $V$ and $S$ can be obtained by [12]:

$$
\begin{aligned}
V & =\frac{4}{3} \pi a b c \\
S & =2 b \sqrt{2} \int_{0}^{\pi} \sqrt{a^{2}+c^{2}+\left(a^{2}-c^{2}\right) \cos (2 \phi)} \sin \phi \\
& \cdot E\left(\frac{c}{b} \sqrt{\frac{2\left(b^{2}-a^{2}\right)}{a^{2}+c^{2}+\left(a^{2}-c^{2}\right) \cos (2 \phi)}} \sin \phi\right) d \phi
\end{aligned}
$$

in which $E=\left(b^{2} \cos ^{2} \theta+a^{2} \sin ^{2} \theta\right) \sin ^{2} \phi$ is a coefficient of the first fundamental form and $\phi$ is a polar angle. Flatness and elongation are defined as $c / b$ and $b / a$, respectively. So, the distribution contours of sphericity and $S / V^{2 / 3}$ (or $a^{\prime}$ ) with different elongation and flatness values can be constructed, as depicted in Figure 4.

Figure 4(a) reveals that if elongation and flatness are both larger than 0.5 , sphericity of an ellipsoid will be close to 1 . $S / V^{2 / 3}$-contours with elongation and flatness are plotted in Figure 4(b). With experimental values of IN and AZ types in Table 1, the hatched regions in Figure 4 can be selected as the optimum solution. The preferred elongation and flatness of an ellipsoid should exceed 0.3 and 0.4 , respectively. This conclusion is comparable with the experimental results on gravel in Figure 4. The selected ellipsoids are thus neither very elongated nor flat.

\section{Shape Simulation of Cement Grains}

Computer X-ray microtomography offers a potential solution for shape assessment of cements, as shown by Garboczi and Bullard [7, 13]. The microstructures of hydrated cements based on actual grain shape and on spherical shape were found significantly different in the study [13]. The results provide an experimental database of this cement that yields some valuable parameters for DEM simulation of cement hydration.

Using cement grains in numerical simulations similar to the real ones would be too expensive. So, it is crucial finding simpler shapes that are sufficiently representative. Similar as in the case of the aggregate simulation, a shape analysis study was therefore conducted with some simpler shapes. Based on this analysis, a simulation strategy is proposed for cement. More realistic but still cost-effective particle shapes are therefore implemented in the DEM system.

Cement is made by raw materials such as limestone and clay, after a process of calcination. Raw cement clinkers consist of hard, large, and roughly rounded particles on a scale of centimeters. Raw cement clinkers are ground in the miller with a small amount of gypsum into small cement grains. Hence, the obtained cement grains will tend to angular shapes on a scale of micrometers. Polyhedra seem therefore more appropriate for simulation of cement grains. Nine polyhedra in Figure 2 are also selected for this shape analysis. As the surface area-to-volume $(S-V)$ relationship was the most important information obtained from the experimental reference [7], values of these shape indices are plotted in Figure 5. The surface to volume relationship can generally be expressed also by $S=a^{\prime} V^{2 / 3}$. Within an effective experimental volume of $10,000 \sim 150,000 \mu \mathrm{m}^{3}$ [7], the coefficient $a^{\prime}=5.8$ was found by regression analysis, with a coefficient of variation lower than 5\%. Proposed shapes should therefore approximate this value. In this case, sphericity can be calculated as 0.83 .

From Figure 5 and Table 2, it is concluded that the heptahedron $I$ and the octahedron $I$ offer the most promising solutions. The octahedron is selected for the simulation study because of allowing an easier transformation into irregular shapes by parameter variation. Three axes can be employed for this purpose, in agreement with [14]. Since the $S$ - $V$ curve of an octahedron is close to that of the reference cement, some limited random variation can be applied accounting for the diversity of particle shape.

Figure 6(a) shows the $S$ - $V$ distributions of randomly generated octahedra with an experimental reference curve. This figure reveals the cases of 1000 particles with longest axis in the $10 \sim 50 \mu \mathrm{m}$ size range. The $S$ - $V$ relationships comply well with the experiments. Figure 6(b) shows an example of a loose packed structure of arbitrary octahedrons supposedly representing fresh cement.

Morphological comparisons can additionally be made between sections of simulated particle structures and of real ones. As an example, Figure 7(a) shows a random section of the simulated structure shown in Figure 6(b) and a $2 \mathrm{D}$ section of a real cement structure obtained by X-ray microtomography. Cement particles in Figure 7(b), displayed in light grey, obviously reveal the angular shape that is similarly revealed by the section of the polyhedral cement particles in Figure 7(a). This adds to the aforementioned 


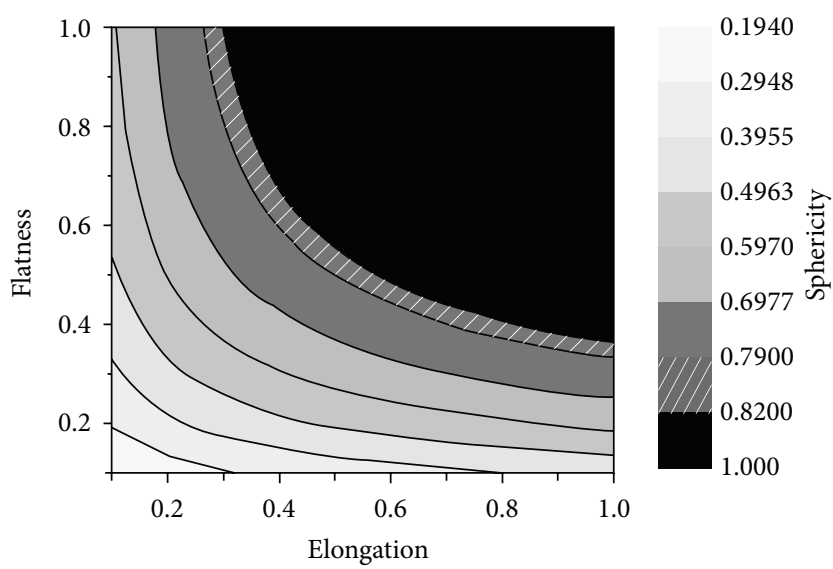

(a)

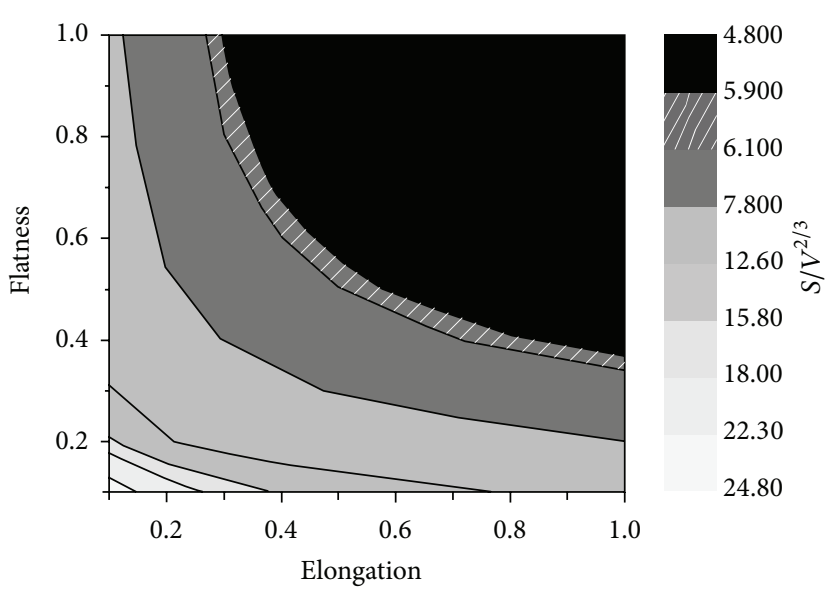

(b)

FIGURE 4: Distribution contours of (a) sphericity and (b) $S / V^{2 / 3}$ with different elongation and flatness of an ellipsoid.

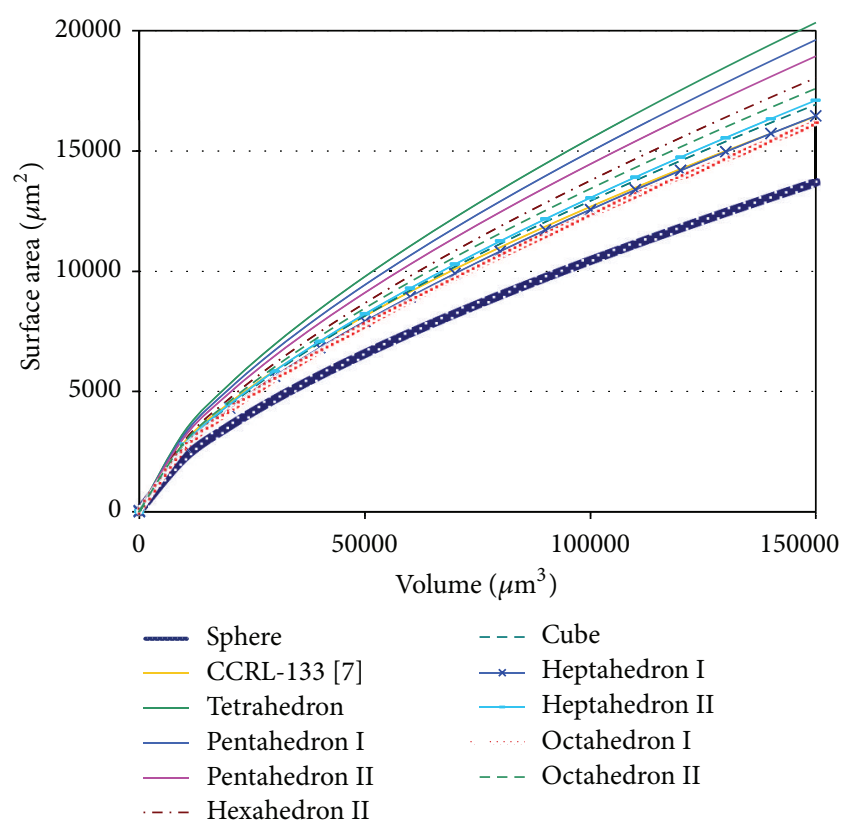

Figure 5: $S-V$ curves of reference cement and some regular polyhedral.

quantitative matching of $S-V$ features of real and simulated cement structures, as shown by Figure 6(a). Therefore, it can be concluded that the proposed simulation method provides a significantly improved option for modelling of cement particles as compared to the conventional approaches in which spheres are employed. This will also have an impact on the hydration process, as well. The simulation of this complicated interference process of hydrating particles is still a demanding issue, however. A possible way out of this hydration simulation problem is to have the outer layer of $\mathrm{C}-\mathrm{S}-\mathrm{H}$ simulated by a dynamic DEM on nanolevel, as is presently developed at Delft University of Technology in the framework of a running Ph.D. study [15].

\section{Discussion and Conclusions}

A particulate material like concrete is composed of irregularly shaped particles, in the form of either aggregate, cement, or mineral admixtures. The definition or simulation of shape is therefore inevitably complex, because it is also intimately connected to that of size. Reconstruction of an irregular shape is technically possible, especially with the development of CT (or $\mu \mathrm{CT}$ ) and $\mathrm{SH}[7,16]$. Even with traditional 3D image analysis, an irregular particle shape can roughly be reconstructed by combining the vertical and horizontal profiles with a 3D mesh program [10, 17]. Examples are plotted in Figure 8. Using a large amount of grains in numerical simulations that have similar shapes as in practice would be too expensive with the eye on equipment and computer time [9]. So, it is crucial finding simpler shapes that are sufficiently representative, since a spherical particle shape as normally assumed in conventional simulation systems can give rise to biased simulation results [13].

Therefore, a shape analysis study was conducted with some simpler shapes for simulation of aggregate as well as of cement. The shape information of real aggregate and cement by CT (or $\mu \mathrm{CT}$ ) was used as references [7, 8]. A simulation strategy based on different origins of arbitrary shaped aggregate grains is proposed. A composite of nine regular polyhedra [11] can properly represent a crushed rock for application in concrete. This method can fully comply with the $S-V$ correlations extracted from experimental findings [8]. Ellipsoids can be used for simulation of gravel of fluvial origin. A kind of relatively flat ellipsoids complies with the $S-V$ information available in experimental investigations [8]. Furthermore, surface texture simulation can also be simply considered by superimposing a sine function on a regular shape [10].

For hydration simulation, a study was conducted pursuing the assessment of physically more realistic shapes than the sphere commonly employed in simulations. This study encompassed polyhedra and ellipsoids. It was shown that a limited variation of octahedra can be considered an 


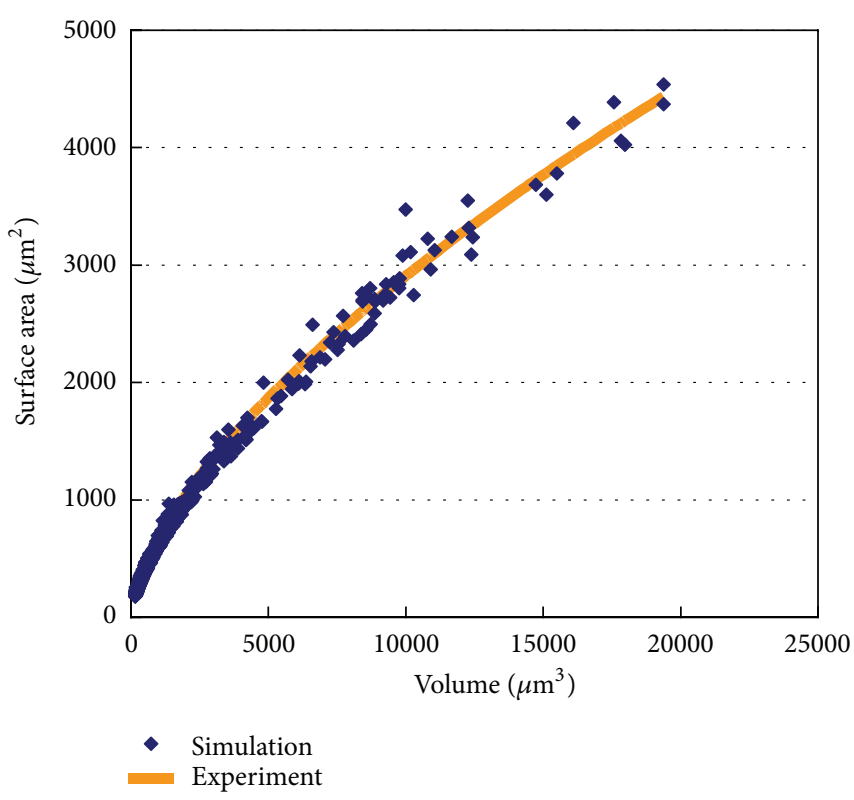

(a)

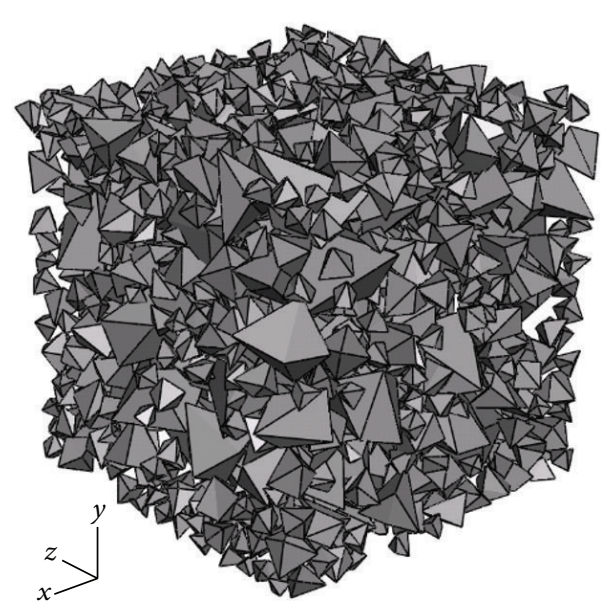

(b)

FIGURE 6: (a) Computer simulation of 1000 particles in the 10 50 $\mu \mathrm{m}$ size range and experimental regression results [7] and (b) visualized structure of compacted grains.

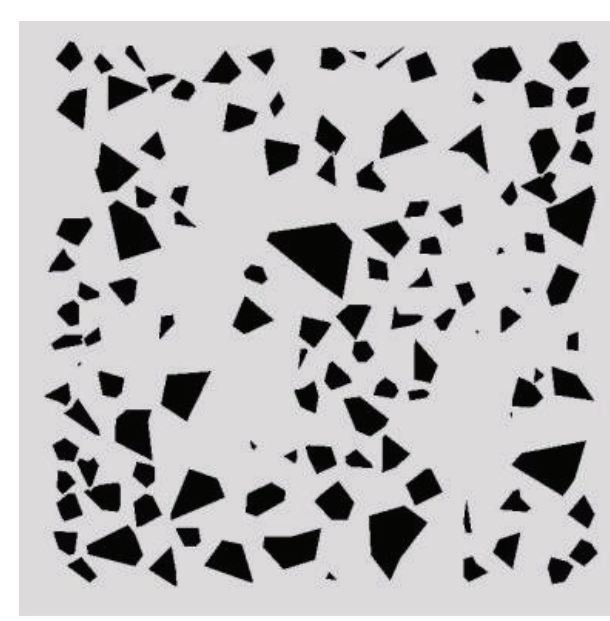

(a)

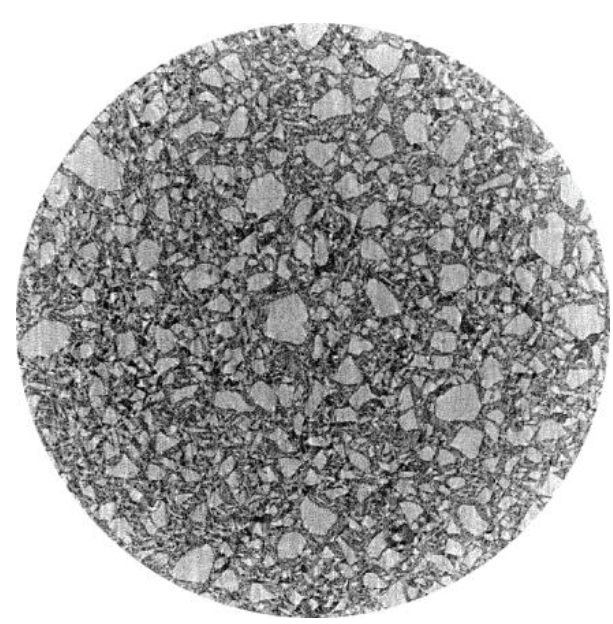

(b)

Figure 7: (a) Section of the simulated structure (1000 octahedron grains in 10 50 $\mu \mathrm{m}$ size range); (b) $2 \mathrm{D}$ section of a real fresh cement structure (Cement-133) obtained by X-ray microtomography; w/c $=0.35$. Source: http://visiblecement.nist.gov/cement.html.
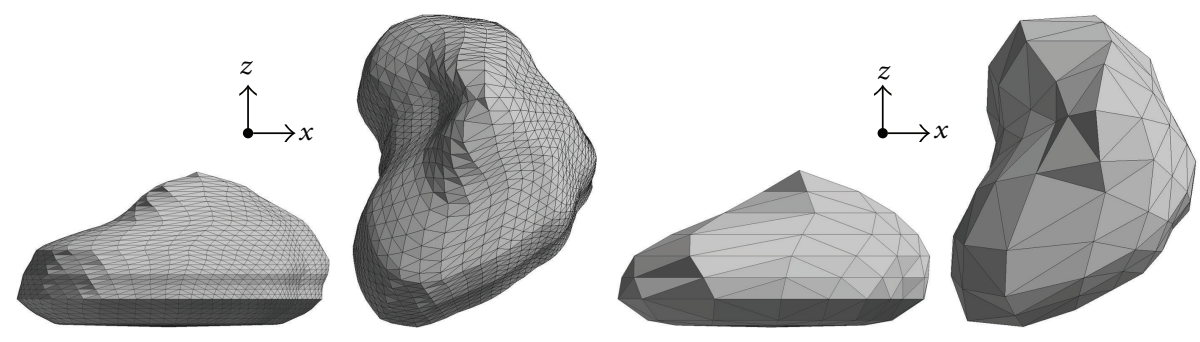

FIGURE 8: Two views of a reconstructed grain with (left) fine 3D mesh and (right) coarse 3D mesh, respectively. 
optimum solution. They offer more realistic $S-V$ relationships as spheres, which is crucial for cement hydration [13] and finally for structure formation governing the pore network system. Morphological comparison shows the similarity of the packed structures of proposed polyhedra with real particles. Implementation of these simulation strategies in the dynamic force-based DEM approach used throughout this study will lead to a more realistic structure of fresh cement paste in concrete technology.

Nowadays, computational concrete, also referred to as "compucrete" or virtual concrete, has been developed for a wide range of research purposes. It provides a proper representation of the heterogeneous concrete material on different levels of its microstructure and renders the possibility of studying the effects of a wide range of technological parameters. Many phenomena in concrete are highly relevant to particle interface properties, for example, interfacial transition zone (ITZ), surface dissolution, and porosimetry. The realistic $S-V$ information incorporated in the DEM approach would yield a better representation of the material and thus forms a more reliable basis for estimating relevant materials properties as compared to systems employing spherical particles.

\section{Conflict of Interests}

The authors declare that there is no conflict of interests regarding the publication of this paper.

\section{Acknowledgment}

The first author is grateful for the continuing support from the National Natural Science Foundation of China (51308013), Beijing Natural Science Foundation (8152009), Specialized Research Fund for the Doctoral Program of Higher Education of MOE (SRFDP, 20131103120022), and the General Program of Science and Technology Development Project of Beijing Municipal Education Commission of China $(004000546314530 / 40)$ for this work.

\section{References}

[1] R. M. German, Particle Packing Characteristics, Metal Powder Industries Federation, Princeton, NJ, USA, 1989.

[2] P. Stroeven, J. Hu, and D. A. Koleva, "Concrete porosimetry: aspects of feasibility, reliability and economy," Cement and Concrete Composites, vol. 32, no. 4, pp. 291-299, 2010.

[3] D. P. Bentz, "Three-dimensional computer simulation of portland cement hydration and microstructure development," Journal of the American Ceramic Society, vol. 80, no. 1, pp. 3-21, 1997.

[4] K. van Breugel, Simulation of Hydration and Formation of Structure in Hardening Cement-based Materials, Delft University Press, Delft, The Netherlands, 1997.

[5] P. Navi and C. Pignat, "Three-dimensional characterization of the pore structure of a simulated cement paste," Cement and Concrete Research, vol. 29, no. 4, pp. 507-514, 1999.

[6] M. Stroeven, Discrete numerical model for the structural assessment of composite materials [Ph.D. thesis], Meinema, Delft, The Netherlands, 1999.
[7] E. J. Garboczi and J. W. Bullard, "Shape analysis of a reference cement," Cement and Concrete Research, vol. 34, no. 10, pp.19331937, 2004.

[8] S. T. Erdogan, P. N. Quiroga, D. W. Fowler et al., "Threedimensional shape analysis of coarse aggregates: new techniques for and preliminary results on several different coarse aggregates and reference rocks," Cement and Concrete Research, vol. 36, no. 9, pp. 1619-1627, 2006.

[9] P. A. Thomas and J. D. Bray, "Capturing non-spherical shape of granular media with disk clusters," Journal of Geotechnical and Geoenvironmental Engineering, vol. 125, no. 2-3, pp. 169$178,1999$.

[10] H. He, Computational modelling of particle packing in concrete [Ph.D. thesis], Delft University of Technology, Ipskamp Drukkers, Delft, The Netherlands, 2010.

[11] W. Guo, "Some material parameters on numerical statistical continuum mechanics of concrete," TU Delft Report 25-88-38, Delft University of Technology, Delft, The Netherlands, 1988.

[12] E. W. Weisstein, Ellipsoid. From MathWorld-A Wolfram Web Resource, http://mathworld.wolfram.com/Ellipsoid.html.

[13] J. W. Bullard and E. J. Garboczi, "A model investigation of the influence of particle shape on portland cement hydration," Cement and Concrete Research, vol. 36, no. 6, pp. 1007-1015, 2006.

[14] H. He, Z. Guo, P. Stroeven, and M. Stroeven, "Discrete element approach to packing of arbitrary shaped particles in concrete," Inżynieria materiałowa, vol. 29, no. 4, pp. 403-407, 2008.

[15] K. Li, M. Stroeven, P. Stroeven, and L. J. Sluys, "C-S-H globule clustering on nano-scale simulated by the discrete element method for pore structure exploration," in Proceedings of the TRANSCEND Conference Water Transport in Cementitious Materials, Guildford, UK, November 2013.

[16] E. J. Garboczi, “Three-dimensional mathematical analysis of particle shape using X-ray tomography and spherical harmonics: application to aggregates used in concrete," Cement and Concrete Research, vol. 32, no. 10, pp. 1621-1638, 2002.

[17] H. He, Z. Guo, P. Stroeven, M. Stroeven, and L. J. Sluys, "Characterization of the packing of aggregate in concrete by a discrete element approach," Materials Characterization, vol. 60, no. 10, pp. 1082-1087, 2009. 

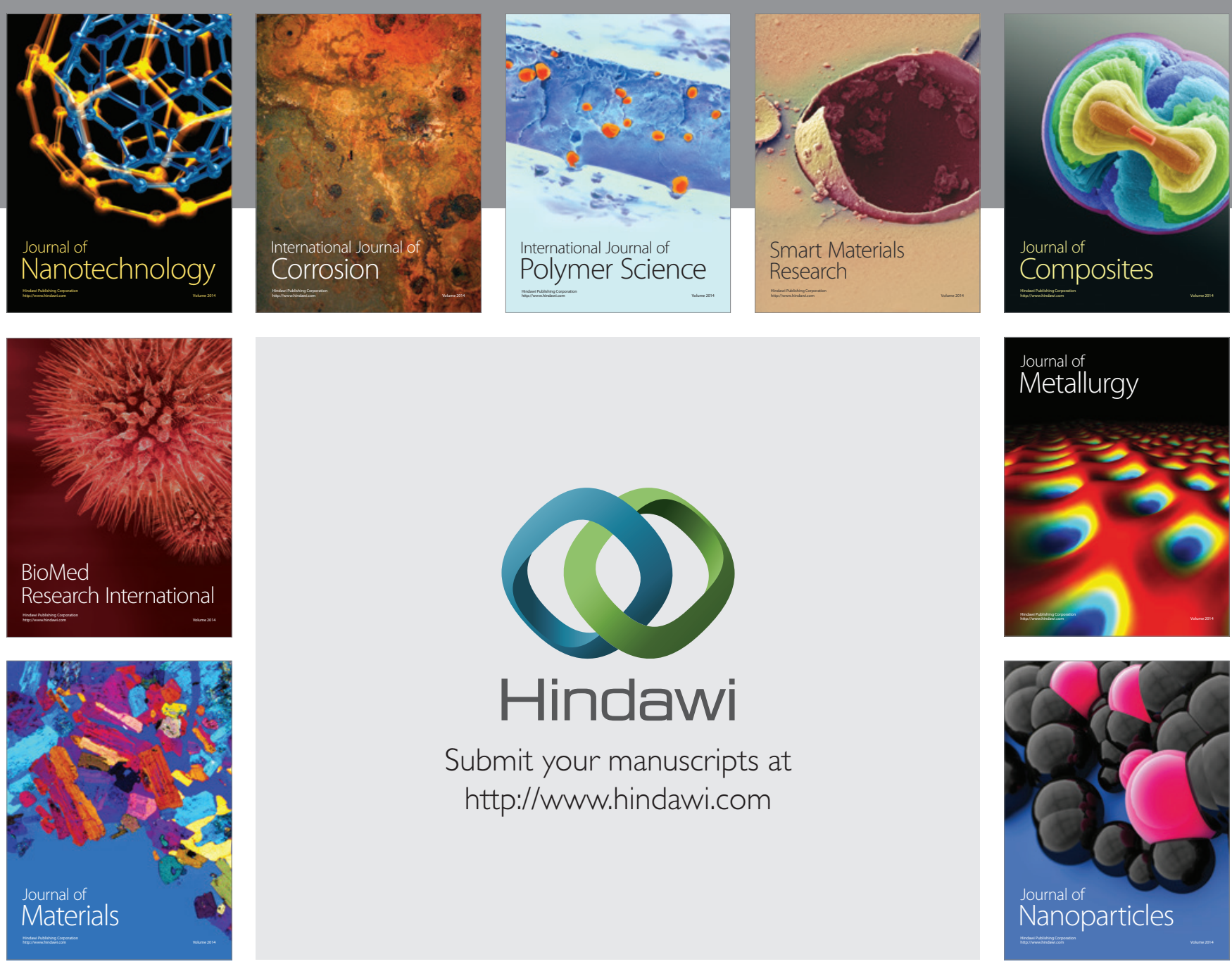

Submit your manuscripts at http://www.hindawi.com
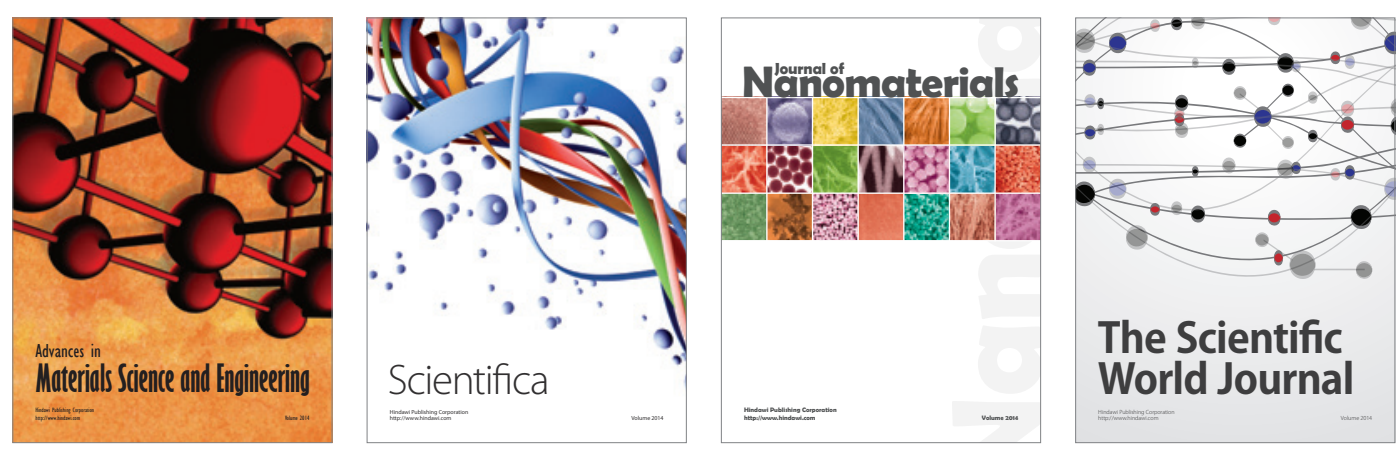

\section{The Scientific World Journal}
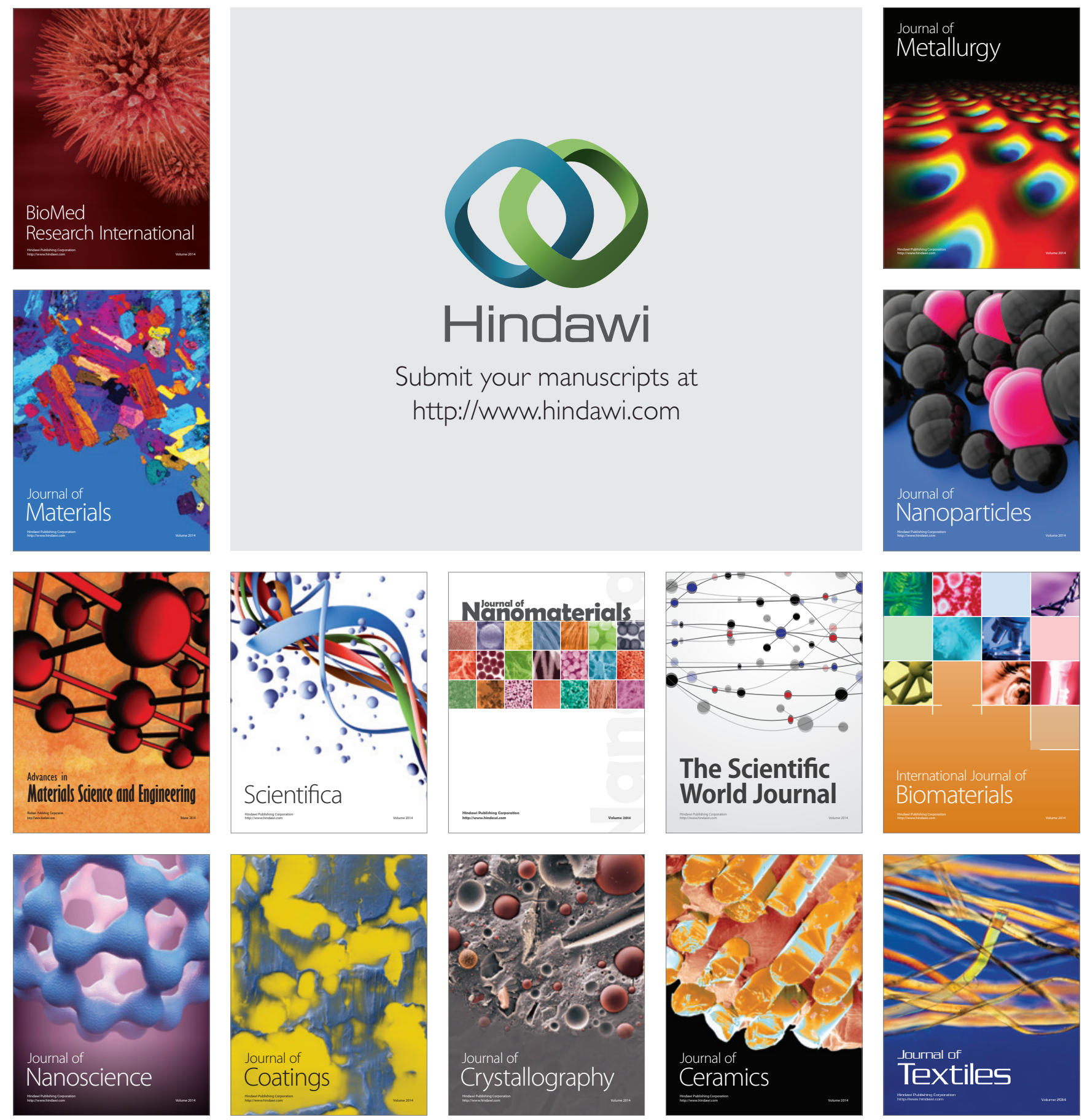